\title{
Proteolytic enzymes and cavitation as strategies to enhanced penetration of drug nanocarriers
}

\begin{abstract}
The poor penetration of drug nanocarriers within tumor tissues is one of the most critical factors which limit their effectiveness. Nanomedicine has developed different strategies in order to overcome this important hurdle. Some of these strategies are based on the degradation of the highly dense extracellular matrix (ECM) which is usually present in many solid tumors. In this sense, one of the most promising approaches consists in the nanoparticle decoration with proteolytic enzymes able to digest the ECM favoring its penetration. Other strategy is based on the capacity of ultrasounds to induce cavitation which propels the nanocarriers to deep areas into the tumor. Both strategies have demonstrated significant improvement in the penetration of nanocarriers in malignant tissues, enhancing their effectivity.
\end{abstract}

Keywords: tumoral tissue penetration, cavitation, proteolytic enzymes, drug nanocarriers, solid tumors, ultrasound
Volume 2 Issue I - 2018

\author{
María Rocío Villegas, ${ }^{1,2}$ Alejandro Baeza, ${ }^{1,2}$ \\ María Vallet Regíl, ${ }^{2}$ \\ 'Department of Chemistry in Pharmaceutical Sciences, \\ University of Central Missouri, Spain \\ ${ }^{2}$ Biomedical Research Center in the Bioengineering, Spain
}

Correspondence: Maria Vallet Regi, Department of Chemistry in Pharmaceutical Sciences, University of Central Missouri, Spain, Email vallet@ucm.es

Received: December 29, 2017| Published: February 09, 2018

\section{Introduction}

Since Matsummura \& Maeda $^{1}$ discovered the tendency of nanocarriers to be extravasated and accumulated in tumoral tissues, nanomedicine has emerged as a promising alternative to conventional cancer therapies. This effect receives the name of Enhanced Permeability and Retention (EPR) effect and is due to the irregular vasculature that irrigated the solid tumor, which present fenestrations of hundreds of nanometers. ${ }^{2}$ This high porosity allows that nanocarriers be selectively accumulated into the diseased tissue. In addition, the high interstitial pressure ${ }^{3,4}$ caused by the deficient lymphatic drainage, favors their retention. However, to reach and to be accumulated into the malignant tissue is only the first step which a nanocarrier must overcome. Once there, they should be capable to achieve a homogeneous distribution within the diseased tissue in order to lead to an effective treatment. Unfortunately, the elevated interstitial pressure and high collagen content ${ }^{5}$ in the ECM suppose a great impediment to their diffusion and provoke that the nanocarriers remain localized in the periphery of the malignancy, which strongly limits their efficacy.

\section{Discussion}

Solid tumors are supported by tumoral extracellular matrix (ECM) which consist in a highly interconnected network of collagen fibers and other components as elastin, proteoglycans, laminin, hyaluronic acid, among other structural proteins. ${ }^{6}$ The interstitial transport, penetration and distribution of macromolecules inside the tissues are governed by two contributions: diffusion and convection. ${ }^{7}$ However, tumoral tissues have high interstitial pressure that hinders the convective transport and limit the transport of macromolecules to diffusion. Moreover, tumoral extracellular matrix present higher collagen contents than healthy connective tissues ${ }^{5}$ and, as the diffusion is inversely depends on the viscosity, the transport of nanoparticles in tumors is much hindered. Thus, the dense collagen networks and its interactions with the rest of structural proteins heighten the greater mechanical stiffness of the tumor and increased the resistance to macromolecules penetration. To improve nanocarriers penetration can be used different types of strategies, one of them consist in the normalization of tumor matrix, that is to say, degrade specifically the components that form the network that support solid tumors, decreasing its stiffness and improve the diffusion of them. Other alternative is the use of ultrasounds; the ultrasound are mechanic waves that can be focused in a local region and induce a propulsion movement of the nanoparticles into the tumor.

Then, the poor penetration of nanocarriers in solid tumors can be addressed by the use of proteolytic enzymes which can be administrated in combination with the drug loaded nanocarrier. As have been mentioned this strategy is based on the capacity of these enzymes to degrade the ECM reducing its density and therefore, favoring the penetration of the nanoparticle into the diseased tissue. As example, use of proteolytic enzymes has demonstrated efficiently digest components of ECM and increase the macromolecule diffusion. Intratumoral injection of enzymes as collagenase, Cathepsin $\mathrm{C}$ and hyaluronidase have demonstrated increase the $10 \mathrm{KDa}$ fluorescent labeled dextran until two folds. ${ }^{8}$ The enzymes can be administered previously to the nanoparticle treatment ${ }^{9}$ or can be anchored ${ }^{10-12}$ on the nanocarrier surface. However, the labile nature of these proteins can compromise their catalytic activity during the journey into the bloodstream. Nanomedicine has provided interesting strategies for protein delivery such as its encapsulation into organic or inorganic shells. ${ }^{13-15}$ This approach increases the circulation time of the housed enzyme at the same time that protects its structure and allows controlling its release in response to the local environment present in the tumor. ${ }^{16}$ Recently, Villegas et al. have reported the synthesis of $\mathrm{pH}$-responsive polymeric nanocapsules able to house collagenase within their matrix and to release it when the system reaches the acidic environment present in the solid tumor (Figure 1). ${ }^{17}$ These nanocapsules allow the controlled and selective release of proteolytic enzymes in the malignant tissue and achieve a significant protection of the enzyme against different insults which can be present in living tissues such as other proteases or elevated temperatures, among others. The same research group has anchored these collagenase nanocapsules on the surface of a drug loaded nanocarrier yielding a significant improvement in the particle penetration and, as a 
consequence of this, higher tumoral cell destruction in comparison with the system without the proteolytic capsules. ${ }^{18}$ This nanocarrier was formed by a mesoporous silica nanoparticle loaded with a potent cytotoxic compound (topotecan) and coated with a lipid bilayer in order to prevent the premature release of the transported drug. The external surface of the lipid bilayer was decorated with an EGFRantibody, able to provoke the selective uptake into lung cancer cells (A549) and collagenase nanocapsules, which provide the capacity to degrade the ECM. This system was evaluated in a 3D tumoral tissue model formed by a cancerous cell monolayer covered with a collagen gel of $200 \mu \mathrm{m}$ of thickness. The collagen matrix contains also malignant cells embedded in order to provide similar rheological parameters that a real tissue. This matrix supposes a strong barrier for particle penetration and it completely hampers the penetration of nanoparticles which did not have collagenase nanocapsules on the external surface whereas, if collagenase nanocapsules are present on the device, the drug nanocarrier induced a significant mortality in the model.



Figure I Use of a) encapsulated proteolytic enzymes or b) cavitation induced by ultrasounds, for improving drug nanocarrier penetration into tumoral tissues.

Other alternative which has been proposed for overcoming the poor nanoparticle penetration is the use of ultrasounds. ${ }^{19}$ Ultrasounds can be focused in deep areas of interest into the body and, in certain conditions, produce cavitation. The cavitation consists in oscillations of a gas bubble in a fluid. ${ }^{20}$ These oscillations can be stable, that it to say, the gas bubbles expand and contract around an equilibrium radius, non-inertial cavitation or, at higher pressure, the gas bubbles can grow unstably during decompression periods and collapse violently during compression periods. This ultimate type of cavitation receives the name of inertial cavitation and is employed to impel the nanocarrier favoring its extravasation and penetration within the diseased tissue (Figure 1). ${ }^{21}$ However, the required pressure can be above clinically acceptable. For this reason, cavitation nuclei such as gas stabilizing solid nanoparticles ${ }^{22,23}$ and micrometric shelled gas bubbles ${ }^{20}$ among others, have been used in order to reduce the pressure required to induce the violent collapse of the gas bubbles. It has been recently reported ${ }^{24}$ in a study where mesoporous silica nanoparticles were propelled by ultrasounds reaching deeper zones of a tumoral tissue model of agarose gel which present similar porosity than endothelial gaps of tumor tissue. For this, channels of $1 \mathrm{~mm}$ of diameter and $50 \mathrm{~mm}$ of length were made in agarose gel and a peristaltic pump remains a constant flow of fluorescent labeled nanoparticles inside them. Then, focus ultrasound was applied in different points of the channel and the penetration of the system was evaluated by fluorescence imaging. The experiment was performed at two frequencies and at different pressure, obtaining better results in the case $1,6 \mathrm{MHz}$ and $4 \mathrm{MPa}$. However, as was mentioned above, the conditions that give raise a successful extravasation and penetration of the nanoparticles into the agarose gel might be too high for the clinical application. Then, the incorporation of micrometric polymeric cups with the nanoparticles was studied. The combination of nanoparticles with cavitation nuclei achieved an efficient extravasation and penetration into the agarose gel decreasing the required pressure by half in order to obtain the same effect when only nanoparticles were employed. Thus, this study validates the possibility to induced nanocarriers extravasation and distribution through the tumor using focused ultrasound at biologically acceptable pressure using combinations of drug nanocarriers and cavitation nuclei.

\section{Conclusion}

Thus, different alternatives have been proposed in order to obtain nanocarriers able to reach deeper areas within tumor tissue. This capacity is indispensable for achieving an effective and uniform nanoparticle distribution throughout the tumoral mass. The use of proteolytic enzymes or ultrasounds would improve the design of new generation of nanosystems enhancing their therapeutic efficacy providing an important progress to their clinical application.

\section{Acknowledgements}

This work has been done thanks to the financial support provided by European Research Council (Advanced Grant VERDI; ERC-2015AdG Proposal No. 694160) and the project MAT2015-64831-R.

\section{Conflict of interest}

The author declares no conflict of interest.

\section{References}

1. Matsumura Y, Maeda H. A new concept for macromolecular therapeutics in cancer chemotherapy: mechanism of tumoritropic accumulation of proteins and the antitumor agent smancs. Cancer Res. 1986;46:63876392.

2. Maeda H, Nakamura H, Fang J. The EPR effect for macromolecular drug delivery to solid tumors: Improvement of tumor uptake, lowering of systemic toxicity, and distinct tumor imaging in vivo. Advanced Drug Delivery Reviews. 2013;65(1):71-79.

3. Khawar IA, Kim JH, Kuh HJ. Improving drug delivery to solid tumors: Priming the tumor microenvironment. J Control Release. 2015;201:7889

4. Nichols JW, Bae YH. EPR: Evidence and fallacy. J Control Release. 2014; 190:451-464.

5. Netti PA, Berk DA, Swartz MA, et al. Role of extracellular matrix assembly in interstitial transport in solid tumors. Cancer Res. 2000;60(9):24972503.

6. Dvorak HF. Tumors: wounds that do not heal. Similarities between tumor stroma generation and wound healing. NEngl J Med. 1986;315(26):1650 1659.

7. Chauhan VP, Stylianopoulos T, Boucher Y, et al. Delivery of molecular and nanoscale medicine to tumors: transport barriers and strategies. Annu Rev Chem Biomol Eng. 2011;2:281-298. 
8. Magzoub M, Jin S, Verkman AS. Enhanced macromolecule diffusion deep in tumors after enzymatic digestion of extracellular matrix collagen and its associated proteoglycan decorin. FASEB J. 2008;22(1):276-284.

9. McKee TD, Grandi P, Mok W, et al. Degradation of fibrillar collagen in a human melanoma xenograft improves the efficacy of an oncolytic herpes simplex virus vector. Cancer Res. 2006;66(5):2509-2513.

10. Parodi A, Haddix SG, Taghipour N, et al. Bromelain Surface Modification Increases the Diffusion of Silica Nanoparticles in the Tumor Extracellular Matrix. ACS Nano. 2014;8(10):9874-9883.

11. Murty S, Gilliland T, Qiao P, et al. Nanoparticles functionalized with collagenase exhibit improved tumor accumulation in a murine xenograft model. Part Part Syst Charact. 2014;31:1307-1312.

12. Kuhn SJ, Finch SK, Hallahan DE, et al. Proteolytic surface functionalization enhances in vitro magnetic nanoparticle mobility through extracellular matrix. Nano Lett. 2006;6(2):306-312.

13. Wen J, Anderson SM, Du J, et al. Controlled protein delivery based on enzyme-responsive nanocapsules. Advanced Materials. 2011;23:4549_ 4553 .

14. Yan M, Du J, Gu Z, et al. A novel intracellular protein delivery platform based on single-protein nanocapsules. Nat Nanotechnology. 2010;5:4853.

15. Gu Z, Yan M, Hu B, et al. Protein nanocapsule weaved with enzymatically degradable polymerie network. Nano Letters. 2009;9(12):4533-4538.

16. Lu Y, Sun W, Gu Z. Stimuli-responsive nanomaterials for therapeutic protein delivery. J Control Release. 2014;194:1-19.
17. Villegas MR, Baeza A, Vallet-Regí M. Hybrid Collagenase Nanocapsules for Enhanced Nanocarrier Penetration in Tumoral Tissues. ACS Appl Mater Interfaces. 2015;7(43):24075-24081.

18. Villegas MR, Baeza A, Noureddine A, et al. Multifunctional Protocells for Enhanced Penetration in 3D Extracellular Tumoral Matrices. Chem Mater. 2017;30(1):112-120.

19. Coussios CC, Roy RA. Applications of Acoustics and Cavitation to Noninvasive Therapy and Drug Delivery. Annu Rev Fluid Mech. 2008;40:395-420.

20. Arvanitis CD, Bazan Peregrino M, Rifai B, et al. Cavitation-enhanced extravasation for drug delivery. Ultrasound Med Biol. 2011;37(11):18381852.

21. Afadzi M, Eggen S, Mørch Y, et al. Multifunctional Nanoparticles for Drug Delivery and Imaging: Effect of Ultrasound on Cellular Uptake and Tumor Tissue Distribution. Ultrasonics Symposium (IUS), IEEE International. Germany; 2012. p. 421-424.

22. Kwan JJ, Graham S, Myers R, et al. Ultrasound-induced inertial cavitation from gas-stabilizing nanoparticles. Phys Rev E Stat Nonlin Soft Matter Phys. 2015;92(2):023019.

23. Kwan JJ, Myers R, Coviello CM, et al. Ultrasound-Propelled Nanocups for Drug Delivery. Small. 2015;11(39):5305-5314.

24. Paris JL, Mannaris C, Cabañas MV, et al. Ultrasound-Mediated Cavitation-Enhanced Extravasation of Mesoporous Silica Nanoparticles for Controlled-Release Drug Delivery. Chemical Engineering Journal. 2018;340:2-8 